(P2-49) Longitudinal Study of Health Conditions After the Sichuan Earthquake in China - The First and Second Year's Results

A. Yamamoto, ${ }^{1}$ X.Y. Hu, ${ }^{2}$ Y. Cheng, ${ }^{2}$ J. Li, ${ }^{2}$ Y. Luo ${ }^{2}$

1. Research Institute of Nursing Care for People \& Community, Akashi, Japan

2. Sichuan, China

Objective: The objective of this study was to identify the daily lives and health conditions of survivors of the Sichuan Earthquake after two years.

Methods: The study was conducted for the survivors $\geq 18$ years of age who experienced the earthquake. The questionnaire included the survivors' socio-demographics, impact of the disaster, and their health status after the disaster.

Results: Approximately 65\% of respondents said that their conditions were healthy in the second year after the earthquake. The changes of daily lives among the survivors were increased from 1st year to 2 nd year, as follows: diet (1st year; 24.5\%-2nd year; $14.1 \%)$, sleep (29.4\%-23.6\%), entertainments $(9.6 \%-15.1 \%)$. As health conditions, the following symptoms were increased; diabetes $(2.8 \%-3.5 \%)$, cardiac disease $(2.9 \%-5.0 \%)$, functional disturbances of body's joints and muscles $(18 \%-21 \%)$. In contrast, the following symptoms were decreased; obesity/skinny (5\%-3.5\%), kidney disease (4.4\%$1.6 \%)$, asthma and allergy (7.2\%-5.6\%). The current health conditions of the survivors included: headache, eyestrain, hand, foot, and/or joint's ache, sleep disorder. With regard to the psychological condition, the mean value of IES-R score was $22.2 \pm 11.8$. Compared with the 1 st year results, the mean value IES-R score significantly decreased (1st year; $30.8 \pm$ $16.8, p<0.001$, by paired t-test).

Conclusions: The survivors' physical and psychological conditions have improved compared to 1 st year results. However most of the survivors still had some health problems in their health. Prehosp Disaster Med 2011;26(Suppl. 1):s152

doi:10.1017/S1049023X11004936

\section{(P2-50) Surviving a Severe Train Crash}

\section{R.M. Forsberg, ${ }^{1}$ B. Saveman ${ }^{2}$}

1. Division of Surgery, Umea, Sweden

2. Department of Nursing, Umea, Sweden

Background: Previous disaster studies mainly focused on analyzing the experience from a psychological or psychiatric perspective. The occurrence of post-traumatic stress disorder also has been the central issue when studying survivors of train crashes. Research concerning being involved in train crashes is scarce.

Objective: The aim of this study was to explore the experience of surviving a severe train crash.

Methods: In 2004, a severe train crash occurred in the south of Sweden. Approximately 78 passengers were on the train. All of them were injured, and two of the passengers died. Fourteen of the survivors agreed to be interviewed five years after the crash. The interviews were narrative, transcribed verbatim, and analyzed using a phenomenological-hermeneutical method. First, a naive reading of the text, later, a structural analysis, and finally a comprehensive understanding in which the other phases were considered and discussed with relevant literature.
Results: The preliminary results show a naive understanding of being in the pre-crash, crash and post-crash phases. Four themes were recognized: (1) life is going on like being on a railway track; (2) the train derails, so do I; (3) back on track again; and (4) life goes on in a new track. The results will be elaborated and discussed together with the comprehensive understanding.

Conclusions: Surviving a severe train crash changes life immediately as well as five years later.

Prehosp Disaster Med 2011;26(Suppl. 1):s152

doi:10.1017/S1049023X11004948

(P2-51) Being Injured in a Cold Environment J. Aléx, B. Saveman

Department of Nursing, Umea, Sweden

Background: Prehospital patients, irrespective of diseases or trauma, might experience discomfort due to a cold environment and are at risk for decreasing body temperature which can increase morbidity and mortality. Research concerning experiences of being injured in a cold environment is scarce.

Objective: The aim of this study was to explore the patients' descriptions of being injured in a cold environment.

Methods: Twenty persons who have been injured in a cold environment during wintertime in the north of Sweden were interviewed; 14 of them were treated with active heat supply. They waited on snow or ice for an ambulance between 8 minutes and 4 hours and 10 minutes. The interviews were performed face-toface and telephone interviews were used when the participants lived far away. The interviews had a storytelling run-up and the participants were asked to narrate the injury event, from just before the event until arrival at the emergency department. The interviews were transcribed verbatim and analyzed by content.

Results: In the preliminary results three themes were formulated: (1) waiting for an ambulance; (2) being cared for; and (3) reflecting on changes in life after the traumatic event. The cold environment influenced the experience of being injured in a negative way. More suffering from the cold than from pain of the injury was described. Those who were treated with active heat experienced it in a positive way.

Conclusions: Discomfort from cold became the largest problem independent of their injury classification. Active heat should be used in prehospital care to reduce the negative experiences from cold.

Prehosp Disaster Med 2011;26(Suppl. 1):s152

doi:10.1017/S1049023X1100495X

(P2-52) Nurse Educators' Experiences of Conducting Trauma Healing to Reduce Anxiety Among Victims of the MT. Merapi Eruption at Universitas Muhammadiyah Yogyakarta

S. Suhariyanto, E. Rochmawati

School of Nursing, Bantul, Indonesia

Background: The number of victims in various refugee camps who experience anxiety after the Mount Merapi eruption. Various factors that cause this condition include a lack of concern for the early treatment of psychological casualties. Support from health professionals, particularly nurses, is needed to prevent the onset of mild anxiety. 
Objectives: The study was carried out in order to help understand the meaning of nurses' experiences of trauma healing to prevent anxiety among the victims of the Mount Merapi eruption.

Method: This was a qualitative research study with a phenomenological approach. In-depth interviews were used to explore participants' experiences conducting trauma healing therapy to the Mount Merapi eruption victims.

Result: The result showed the implementation of trauma healing therapy includes: (1) assessment of emotional responses; (2) physical examination; and (3) psychological assistance. The therapy being implemented includes: (1) five-finger hypnosis therapy; (2) stopped thinking therapy; and (3) progressive relaxation. The impacts of trauma healing included: (1) spirit returning; (2) increased of relaxation; (3) calmness; (4) normal vital signs; and (5) the ability to interact with other refugees. Problems experienced included the large number of refugees, the noisy environment, and a lack of concentration from the nurse when providing therapy.

Conclusion: To solve the problem, nurses are expected to recognize the response required for the victim and apply interventions based on the assessment, data analysis, planning, implementation, and evaluation. Victims of the Mount Merapi eruption are expected to attempt to apply the self-trauma healing.

Prehosp Disaster Med 2011;26(Suppl. 1):s152-s153

doi:10.1017/S1049023X11004961

(P2-53) Investigation of Comprehension of Disaster/ Emergency Nursing in Nurse Practitioners in Ningbo Area, China

X.W. Wang, J. Xu, Y.J. Shen

Department of Nurse Administration, Ningbo, China

Background: Knowledge of disaster/emergency nursing is essential to nurse practitioners (NP) due to the increasing frequency of disasters. The objective of this study was to identify the knowledge and the response relating $\mathrm{D} / \mathrm{EN}$ and to investigate whether the reactions vary among NPs in different level hospitals in Ningbo Area, China.

Methods: Pre- and post-test questionnaires were used. A total of 297 NPs in five hospitals (two tertiary hospitals, two secondary hospitals, one primary hospital) were involved in this study. Five lectures were implemented based on disaster/emergency nursing from 8 December 2009 to 14 April 2010.

Results: The mean age of the sample was 31.54 years. Average working experience was 9.57 years. Only 12 participants from the emergency department attended the lecture, yet the lecture was advertised one week in advance. The mean score was 8.88 (pre-test), 12.97 (post-test). Most of the low scores $(<7)$ on the pre-test were from primary hospitals (43\%), while only $0.6 \%$ of staff from tertiary hospitals scored that low. A total of $45 \%$ of NPs failed to distinguish the contribution of different zones of triage. Nearly $24 \%$ of participants considered that the frequency of ALS training should no more than once a month. All participants comprehended the contribution of a triage system after attending lecture, $35 \%$ of NPs received perfect scores on the post-test.

Conclusions: Comprehension of disaster/emergency nursing in NPs in Ningbo is deficient. Primary hospital NPs had a lower score than high-level hospitals. Education and training programs associated with disaster/emergency nursing are necessary for NPs.

Prehosp Disaster Med 2011;26(Suppl. 1):s153

doi:10.1017/S1049023X11004973

(P2-54) Legislation Shaped by an Emergency: Methanol Poisoning Experience at Kenyatta National Hospital, Kenya

A.W. Akida, ${ }^{1}$ M. Isinta, ${ }^{2}$ F.O. Ndiawo, ${ }^{1}$ D. Agedo, ${ }^{2}$

J. Tsinanga ${ }^{2}$

1. Accident and Emergency, Nairobi, Kenya

2. Nairobi, Kenya

Introduction: Methanol poisoning is an uncommon medical emergency linked with consumption of traditional brews made with methanol and formalin and associated with high-mortality rates.

Objectives: Healthcare workers will review the latest worldwide trends of methanol poisoning cases, explain the factors perpetuating methanol poisoning in Kenya, describe the pathophysiological concepts associated with methanol intoxication, and discuss the latest measures to combat methanol poisoning in Kenya and their worldwide applicability.

Background: Methanol intoxication is an acute illness resulting from consumption of toxic quantities of methanol. The largest tragedy occurred in September 2006 in Nicaragua. A total of 800 fell ill, 46 were killed. In the US, the last incidence was in 1951. Cases were reported in Africa, Tunisia, Tanzania, Uganda, but Kenya, it runs the most rampant. The majority of victims (79\%) are young males, (22-30 years of age). Most are single, childless, and have a low-educational status. Motivating factors for intoxication include stress, idleness, peer-pressure, availability of alcohol, and curiosity.

Pathophysiology: Toxicity results from liver enzymatic metabolism of methanol to formaldehyde and formic acid causing severe metabolic acidosis. Common features include inebriation, abdominal pains, bilateral blindness, and complications, including severe renal failure and death. The goals of management include comprehensive assessment, laboratory works, and radiography. Ethanol, fomepizole, and folate are the all-important antidotes.

Recommended Measures: Kenyatta National Hospital, the main recipient of these emergencies established emergency measures other than public awareness campaigns. Nationally, policies embrace an inter-sectoral Approach - Medical Services and Public Health Ministries will avail resources and build health worker capacity in research and continuous education. Recently, local brews were legalized through the Alcoholic Drinks Control Act 2010 for quality control. Ministries of Education and Youth Affairs will coordinate and initiate youth development and support programs to create employment.

Prehosp Disaster Med 2011;26(Suppl. 1):s153 doi:10.1017/S1049023X11004985

(P2-55) The Role of Nursing in International Disasters: Lessons Learned

S.M. Burke, ${ }^{1}$ S. Briggs ${ }^{2}$

1. Nursing, Boston, United States of America

2. Surgery, Boston, United States of America

A disaster may result from a serious or sudden catastrophic event that has the potential for massive loss of infrastructure and 